\title{
Author Correction: Abundance and diversity of the faecal resistome in slaughter pigs and broilers in nine European countries
}

Patrick Munk (D), Berith Elkær Knudsen, Oksana Lukjancenko, Ana Sofia Ribeiro Duarte, Liese Van Gompel, Roosmarijn E. C. Luiken, Lidwien A. M. Smit, Heike Schmitt, Alejandro Dorado Garcia, Rasmus Borup Hansen, Thomas Nordahl Petersen, Alex Bossers (D), Etienne Ruppé, EFFORT Group, Ole Lund, Tine Hald, Sünje Johanna Pamp, Håkan Vigre, Dick Heederik, Jaap A. Wagenaar, Dik Mevius and Frank M. Aarestrup (1)

Correction to: Nature Microbiology https://doi.org/10.1038/s41564-018-0192-9, published online 23 July 2018.

In the version of this Article originally published, the surname of author Oksana Lukjancenko was spelt incorrectly as 'Lukjacenko'. This has now been corrected. 